\title{
LA IMPORTANCIA DE LA APLICACIÓN DE LAS LEYES Y NORMATIVAS DE SEGURIDAD E HIGIENE OCUPACIONAL PARA DISMINUIR RIESGOS LABORALES EN SOLCA
}

\author{
AUTORES: Maryuri Yahaira Briones Mera ${ }^{1}$ \\ Fidel Alfredo Chonillo Toala ${ }^{2}$ \\ Jose Felix Peñafiel Loor ${ }^{3}$ \\ Freddy Fernando Ponce Toala ${ }^{4}$
}

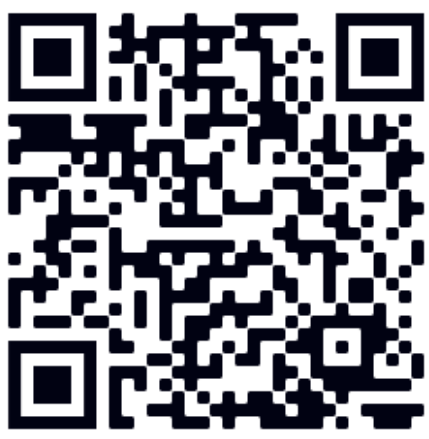

DIRECCIÓN PARA CORRESPONDENCIA:Josymar_86@yahoo.es

Fecha de recepción: 04-10-2019

Fecha de aceptación: 10-11-2019

\section{RESUMEN}

Una política de seguridad y salud en el trabajo es la declaración del grado de compromiso, en función del tipo de empresa u organización, teniendo en cuenta el nivel de exposición a peligros y riesgos de los trabajadores este estudio sobre la seguridad e higiene ocupacional y su incidencia en los riesgos laborales, tiene como objetivo verificar el cumplimiento de la normativa de seguridad e higiene ocupacional y su incidencia en los riesgos laborales. Para la realización del diseño metodológico se utilizó el método inductivo, deductivo y estadístico, dentro de este marco las técnicas utilizadas fueron: observación, encuesta y entrevista cuyos procedimientos metodológicos permitieron la captación de información, además de estas técnicas se sintetizo en la búsqueda de información relevante que permitió cristalizar este resultado; además se detallan los recursos materiales y humanos, luego del cual se realizan las conclusiones

PALABRAS CLAVE: Capacitación, Observación, Seguridad, Recursos, Riesgos

\section{THE IMPORTANCE OF THE APPLICATION OF THE LAWS AND REGULATIONS OF OCCUPATIONAL SAFETY AND HYGIENE TO REDUCE LABOR RISKS IN SOLCA}

\section{ABSTRACT}

A safety and health at work policy is the declaration of the degree of commitment, depending on the type of company or organization, taking into account the level of exposure to hazards and risks

\footnotetext{
1 Ing. Maryuri Yahaira Briones Mera Universidad Particular San Gregorio de Portoviejo E-mail: Josymar_86@yahoo.es

2 Universidad Estatal del Sur de Manabí Servidor Público E-mail: achonillo2017@ @otmail.com

${ }^{3}$ Universidad Estatal del Sur de Manabí campus los Ángeles Docente de la Carrera de Auditoria E-mail: jpfelix@hotmail.es

${ }^{4}$ Universidad Estatal del Sur de Manabí campus los Ángeles Servidor Público Dpto. de Investigación E-mail: fponcetoala@yahoo.es
} 
Maryuri Yahaira Briones Mera, Fidel Alfredo Chonillo Toala, Jose Felix Peñafiel Loor...

of workers this study on occupational safety and hygiene and its incidence in occupational risks, aims to verify compliance with safety and occupational hygiene regulations and their impact on occupational risks. To carry out the methodological design, the inductive, deductive and statistical method was used. Within this framework, the techniques used were: observation, survey and interview, whose methodological procedures allowed for the collection of information, in addition to these techniques, it was synthesized in the information search relevant that allowed this result to crystallize; In addition, the material and human resources are detailed, after which the conclusions are made.

KEYWORDS: Training, Observation, Security, Resources, Risks

\section{INTRODUCCIÓN}

En la actualidad, en el Ecuador es necesario que las empresas e instituciones públicas y privadas cuenten con normativas regidas a las leyes vigentes del país para que sus trabajadores cuenten con protección para obtener condiciones óptimas las mismas que garantizaran una buena seguridad para proteger la salud y conservar la higiene laboral en los trabajadores.

Dentro del desarrollo empresarial, industrial del mundo moderno, es necesario que las empresas cumplan con las disposiciones y leyes universales tanto de la Organización Internacional del Trabajo (OIT), Organizacional Mundial de la Salud, así como los reglamentos y políticas que rigen la actividad empresarial en el Ecuador a través de diversidad de normativas que garanticen el bienestar de los empleados en el medio laboral disminuyendo riesgo laborales y amenazas a la salud relacionadas con su área de trabajo.

La seguridad e higiene ocupacional al ser una actividad técnico administrativa va encaminada a prevenir la ocurrencia de accidentes y enfermedades, cuyo resultado final es el daño que a su vez se traduce en pérdidas.

Por eso, para analizar esta problemática es necesario mencionar sus causas. Una de ellas es la ausencia de capacitaciones en temas de seguridad e higiene ocupacional, lo cual hace que los trabajadores no desempeñen su labor de manera cómoda y segura, ocasionando enfermedades o accidentes en su lugar de trabajo.

\section{DESARROLLO}

\section{JUSTIFICACIÓN}

La realización de este estudio de seguridad e higiene ocupacional está dirigida a diagnosticar, evaluar y analizar el conocimiento de normativas de seguridad establecidas por la OIT Y OMS, por cada riesgo hallado en SOLCA Jipijapa se tomará en cuenta, y de esta manera se encontrará las áreas más vulnerables a daños a la salud de los empleados.

A través de esta investigación, en la cual se pretende fomentar la prevención de riesgos en la Sociedad de Lucha Contra el Cáncer Jipijapa, de manera que se logre detectar y evitar los accidentes de trabajo y enfermedades profesionales, facilitando la ejecución del trabajo en condiciones confiables al verificar el cumplimiento de los reglamentos de la sociedad relacionaos con la auditoria SHO. 
Cabe señalar que, la siguiente estudio de seguridad e higiene ocupacional busca el mejoramiento de las políticas de prevención de riesgos laborales identificando la existencia de peligros a las condiciones físicas y operativas de labor en las funciones diarias de los empleados.

El motivo es que a través de la indagación se alcance el conocimiento sobre normativas dirigidas a la seguridad e higiene ocupacional, para que pongan en práctica en cada área de trabajo acorde a las sugerencias y a su vez contribuyendo a la diminución de riesgos.

\section{CUERPO DEL ANALISIS}

Los sistemas de seguridad y medidas preventivas de accidentes y enfermedades ocupacionales forman parte de normativas políticas y legales emitidas por la Organización Mundial del Trabajo, la cual involucra la disminución de sucesos que desencadenan los sobrecostos de accidentes en el lugar de trabajo como la indemnización a causa de lesiones.

Siendo un centro oncológico, esta entidad privada encargada de prevenir, diagnosticar y promover tratamientos contra el cáncer, descubriendo a tiempo enfermedades cancerígenas, su personal está bajo los efectos de reactivos para la extracción de muestras infecciosas y patológicas, generando riesgos laborales, es normal que en esta institución en la que por su naturaleza existen riesgos y peligros tanto físicos como ambientales, entre lo que tenemos:

Se considera la existencia de políticas internas de seguridad, salud laboral y gestión ambiental de la entidad en el cual se sumen varios compromisos con el fin de asegurar la protección del personal que labora en la institución, se debe establecer normativas acordes a las normas establecidas por la OIT y OMS, para que la sociedad disminuya y controle de esa manera los riesgos que afectan a la salud del empleado.

"La jefatura de servicios de seguridad e higiene en el trabajo, es el área del instituto cuyo objetivo primordial es promover la prevención de riesgos del trabajo mediante actividades, programas y campañas que deben realizar las dependencias y entidades afiliadas al régimen de la ley, a fin de garantizar la seguridad y salud de los trabajadores, así como nombran, supervisar y evaluar el proceso de dictaminarían de riesgos de trabajo e invalidez". Según la (Instituto de Seguridad y Servicios Sociales de los Trabajadores del Estado, 2018)

Lo principal de la seguridad e higiene ocupacional es la prevención de riesgos creando estrategias, métodos y programas que sean acordes a lo establecido en la ley como la OIT y OMS conlleva a la disminución de daños a la salud de cada trabajador dependiendo su lugar de trabajo y de esta manera creando un mejor ambiente laboral.

"La seguridad y la salud laboral engloba a todas aquellas acciones que se llevan a cabo para poder garantizar que los trabajadores desarrollen sus tareas con el mínimo de riesgo posible. Estas medidas vienen determinadas por la Organización Internacional del Trabajo, la OIT. Que es el organismo internacional que se encarga de establecer dichas condiciones de trabajo en todo el mundo". Para (Marben Abogados, 2015)

En la actualidad se han creados medidas preventivas como lo establece la OIT el cual es uno de los organismos encargado de que toda entidad cumpla con los reglamentos o normas de seguridad e higiene ocupacional para sus empleados, para que el trabajador no esté tan expuesto a los riesgos imperceptibles en su lugar de trabajo y así crearle un mejor clima laboral.

Dentro de la Finalidad de la Seguridad e higiene ocupacional, "El fin de la higiene ocupacional es proteger la salud del trabajador, con el objetivo de optimizar su labor y el desarrollo profesional 
Maryuri Yahaira Briones Mera, Fidel Alfredo Chonillo Toala, Jose Felix Peñafiel Loor...

dentro del ambiente laboral. Por ende, en términos generales, este concepto se refiere a una técnica de prevención de enfermedades en el trabajo". Según (Organización Internacional del Trabajo, 2014)

La importancia de la finalidad de SHO es que se cumplan con las funciones o normativas de salvaguardar la salud, optimizando su trabajo o generan dándole un mejor ambiente laboral con la disminución de riesgo procedentes del trabajo al cual ellos se dedican.

La higiene ocupacional, "Es la ciencia de la anticipación, identificación, evaluación y control de los factores de riesgo que se originan en el lugar de trabajo o en relación con él y que pueden poner en peligro la salud y el bienestar de los trabajadores, teniendo también en cuenta su posible repercusión en las comunidades vecinas y en el medio ambiente en general. (Rodriguez, 2010)

Es aquella que depende del ambiente laboral en el que los trabajadores desarrollan sus actividades, es decir la ciencia que se encarga de evaluar y controlar los factores de riesgos los cuales pueden ocasionar enfermedades y afectar la salud de los miembros de la institución.

"La seguridad y salud ocupacional aplicadas a los centros de trabajo consiste en salvaguardar la vida y preservar la salud y la integridad física de los trabajadores por medio del dictado de normas encaminadas tanto a que les proporcionen las condiciones para el trabajo, como a capacitarlos y adiestrarlos para que se eviten dentro de lo posible las enfermedades y los accidentes laborales". (Navarrete, 2013)

Por tanto, la seguridad e higiene ocupacional es la disciplina de un conjunto de técnicas que ayudan a evaluar y controlar los factores físicos y psicológicos a los cuales se encuentran expuestos los trabajadores en sus lugares de trabajos y puedan deteriorar la salud y causar enfermedades. Todas las instituciones sean públicas y privadas deben formar al personal mediante capacitaciones referentes a seguridad e higiene ocupacional para así evitar posibles accidentes y enfermedades laborales.

Los Objetivo de la Seguridad e higiene ocupacional, "Procurar que a lo largo de toda su vida de trabajo el personal se vea libre de cualquier daño a su salud e integridad física ocasionado por las sustancias que manipulan; los equipos, maquinarias o herramientas que utilizan; o por las condiciones en que desarrollan sus actividades. En igual forma, intenta garantizarles un ambiente agradable y exento de incomodidades. (Haddad, 2013)

El objetivo principal de la seguridad e higiene ocupacional es salvaguardar la integridad física y psicológica de los trabajadores en sus puestos de trabajo es decir que ellos se vean libre de cualquier peligro que afecte su integridad en el desarrollo de sus actividades a lo largo de toda su vida.

Su Importancia de la Seguridad e higiene ocupacional en el ámbito de trabajo indica, La seguridad e higiene ocupacional en el trabajo son aspectos que deben tenerse en cuenta en el desarrollo de la vida laboral de la empresa, esa es su importancia. Su regulación y aplicación por todos los elementos de la misma se hace imprescindible para mejorar las condiciones de trabajo. Aunque su conocimiento en profundidad sea necesario para los trabajadores, cobra un especial interés en los mandos responsables de las empresas ya que de ellos se exige lograr la máxima productividad sin que ello ponga en peligro vidas humanas o pérdidas en materiales y equipos.

El enfoque técnico-científico da una visión de conjunto de la seguridad y la higiene en la empresa siguiendo técnicas analíticas, operativas y de gestión es símbolo de desarrollo. "Los responsables de la seguridad e higiene deben saber que hacer en cada caso, cómo hacerlo, y cómo conseguir que 
lo hagan los demás y, sobre todo, que se haga bien - calidad. Una buena prevención de los riesgos profesionales, basados en un profundo conocimiento de las causas que los motivan y en las posibilidades que hay a nuestro alcance para prevenir los problemas, evitará consecuencias muy negativas para el perfecto desarrollo de la vida laboral". (Velasco, 2015)

Es muy importante que toda institución independientemente de su tamaño garantice a los trabajadores un ambiente de trabajo seguro, libre de accidentes y enfermedades que pueden ocasionar un deterioro en su salud. Además, es de vital importancia que se adopten medidas de seguridad en los centros de trabajo para proteger a la mayor riqueza que toda organización debe de considerar, los trabajadores.

La Inspección de Seguridad laboral, Se basa que "La inspección de seguridad es una técnica analítica de seguridad que consiste en un análisis, realizado mediante observación directa de las instalaciones, equipos y procesos productivos (condiciones, características, metodología del trabajo, actitudes, aptitudes, comportamiento humano) para identificar los peligros existentes y evaluar los riesgos en los diferentes puestos de trabajo. (Asociacion Española para la Calidad, 2013)

La Inspección de seguridad es una técnica preventiva que está dirigida hacia técnicas generales de seguridad previas a que ocurra el accidente y es considerada también como una técnica analítica puesto que tiene como objetivo detectar más que corregir conductas y actuaciones que generen peligro. Esta inspección de seguridad se realiza de una manera exhaustiva en todos los equipos, procesos e instalaciones que estén en funcionamiento en conjunto con los responsables de cada área relacionada al trabajo.

La Inspección de Seguridad es la técnica analítica previa al accidente/incidente más conocida y practicada como medio para detectar los peligros y controlar los Riesgos que puedan afectar a las personas o a la propiedad y se considera fundamental dentro de cualquier programa de Prevención, por sencillo que éste sea. Permite estudiar las condiciones de seguridad en las instalaciones y actuaciones en los puestos de trabajo. (Prado, 2012).

A través de las inspecciones de seguridad se podrán identificar y detectar los peligros de accidentes, de las enfermedades y de aquellas disfunciones del empleado que pueden ocasionar perdidas de cualquier índole, para posteriormente corregirlos. Es de carácter preventivo, ya que se debe realizar antes de que se presente la pérdida o daño, para poder tomar las medidas necesarias que impidan desarrollar la potencialidad negativa de aquellos peligros que en ella han sido detectados.

Al mismo tiempo la Higiene laboral "La higiene laboral está relacionada con las condiciones ambientes de trabajo que garantice la salud física y mental, y con las condiciones de bienestar de las personas". Para (Hichez, 2013)

La higiene en el lugar laboral es importante para que los trabajadores cuenten con un ambiente estable que no los afecte mental ni físicamente, para que se sienta cómodo en un entorno de bienestar que le genera la empresa.

La Seguridad e Higiene del trabajo como disciplina técnica. "Para poder desempeñar su función el técnico de seguridad o ingeniero de seguridad debe poseer conocimientos de los procesos tecnológicos, ya que solo a partir de estos podrá llegar a analizar los riesgos inherentes a cada etapa del proceso y estudiar las medidas de prevención a adoptar, procurando su inclusión en la fase más temprana del proceso, en el proyecto". Según (Cortes, Seguridad e higiene en el trabajo: tecnicas de prevencion de riesgos laborales, 2007, pág. 45) 
Maryuri Yahaira Briones Mera, Fidel Alfredo Chonillo Toala, Jose Felix Peñafiel Loor...

Para que el profesional pueda desempeñar las funciones con normalidad debe tener conocimientos de seguridad e higiene ocupacional, ya que así el podrá analizar bien los riesgos acordes a las etapas correspondientes y mediante esto creara norma de prevención a los trabajadores de la institución para evitarle daños a la salud y mejorando su clima laboral.

Por lo tanto a la Técnica de seguridad "Para conseguir el objetivo concreto de la seguridad: detectar y corregir los diferentes factores que intervienen en los riesgos de accidentes de trabajo y controlar sus consecuencias, la seguridad se sirve de unos métodos, sistemas o formas de actuación definidas, determinadas técnicas de seguridad

En la lucha contra los accidentes de trabajo podemos actuar de diferentes formas, dando lugar a las diferentes técnicas, dependiendo de la etapa o fase del accidente en que se actúe:

Análisis del riesgo (identificación del peligro y estimación del riesgo).

Valoración del riesgo.

Control de riesgo". (Cortés, 2007, pág. 106)

Para conseguir una buena seguridad se debe aplicar los distintos reglamentos que rigen y están aprobados por los organismos internacionales, así como como por el estado ecuatoriano y poder minimizar los accidentes laborales los cuales traen consecuencias a la salud y un bajo rendimiento y de esta manera determinar métodos para el control de riego.

La importancia de las disciplinas asociadas a la seguridad e higiene laboral. Se basa "Las actualizaciones en materia de prevención inherentes a seguridad e higiene laborales, se hallan enmarcadas por un carácter multidisciplinario e interdisciplinario y por lo tanto, en su necesaria integración en todas las fases del proceso productivo y organizativo de la institución". Para (Higiene y Seguridad Laboral, 2011).

En la actualidad las disciplinas asociadas a la seguridad de higiene ocupacional son encuadradas a la forma multidisciplinaria e interdisciplinaria lo cual genera la necesidad de la unión y complementación de fases que generen una capacidad de trabajo en la empresa.

En relación a los Riesgos laborales y daños derivados del trabajo. Se establece que "Se consideran daños derivados del trabajo a las enfermedades, patologías o lesiones producidas con motivos u ocasión del trabajo. Se trata de lo que en términos más comunes o tradicionalmente se habla como enfermedades o patologías laborales o accidentes laborales, aunque con un sentido más amplio y menos estricto. Es decir, cualquier alteración de la salud, incluidas las posibles lesiones, debidas al trabajo realizado bajo unas determinadas condiciones" (Grau, 2000).

En las empresas existen y con el paso del tiempo se generan diferentes riesgos que afectan a la salud del trabajador ya que no tienen conocimiento de los daños que le pueden generar por causa del trabajo que desempeñan, creándoles lesiones por no tener los implementos necesarios y enfermedades laborales por el mal ambiente laboral.

\section{DESEMPEÑO LABORAL}

"El desempeño laboral, contempla los procesos a los cuales se someten los recursos humanos, considerando diferentes métodos y criterios para la medición del rendimiento de los trabajadores: 1) Planeación de Recursos Humanos. Al evaluar los recursos humanos de una compañía, debe disponerse de datos que describan el potencial general y de promoción de todos los empleados, especialmente, de los ejecutivos clave" (Mondy, Noe, \& Quiñones, 2005) 
El desempeño del trabajador se mide con conocimientos y criterios que contemplen al rendimiento de cada uno, ya que recursos humanos es el encargado de evaluar el potencial y la metodología que tiene empleado para cumplir con su trabajo sin ninguna interrupción.

Los Elementos que influyen en el desempeño laboral Según estudiosos es "Diversos autores han encontrado diferentes variables para evaluar el desempeño de las personas en sus trabajos, tal es el caso de Davis y Newtrons, quienes aseguran que el desempeño laboral se ve afectado por factores como: capacidades, adaptabilidad, comunicación, iniciativa, conocimiento, trabajo en equipo, estándares de trabajo, desarrollo de talentos, potencia el diseño del trabajo, maximizar el desempeño" (Navarro, 2014)

Los principales elementos que son importante para que el trabajador se sienta bien en su lugar de trabajo son saber sus capacidades, conocer si se adapta a cualquier ambiente, que tenga fluidez al momento de comunicarse, quiera superarse, obtenga conocimientos del trabajo que va a desempeñar, que le guste trabajar en equipo, que maneje diferentes métodos de trabajo que puedan ayudar al desarrollo de la empresa.

Locke define la satisfacción laboral como un estado emocional positivo o placentero resultante de la percepción subjetiva de las experiencias laborales del sujeto o como repuesta afectiva de una persona a su propio trabajo. (Chiang \& San Martín, 2015)

Se considera que la satisfacción es medida por el estado emocional o que el ambiente de trabajo de los empleados sea el adecuado para que ellos tengan resultados favorables al momento de realizar su tarea laboral ya que sienten que es el mejor ambiente lo cual les genera aplacer realizar sus labores.

\section{METODOLOGIA O DISEÑO METODOLOGICO}

Metodología analítica: Se utilizó este método de investigación el cual nos permitió estudiar la realidad mediante la ejecución de las encuestas a los empleados de la Sociedad de Lucha Contra el Cáncer.

Metodología descriptiva: Este método de investigación se utilizó para poder detallar cada proceso describiendo todos los riesgos y las actividades que los empleados de la Sociedad de Lucha Contra el Cáncer realizan.

Método bibliográfico: Este método nos permitió sintetizar los diferentes aspectos conceptuales teóricos, relacionadas con las variables de la investigación.

Método estadístico: Fue utilizado para que nuestra investigación obtenga enfoques estadísticos a través de resultados los cuales se manifiestan en gráficos, en el proceso de recolecta y se pone la información en porcentajes cuyos niveles serán de mínimo a máximo.

Inductivo: Se utilizó en esta investigación este método para observar los riesgos a los que están expuesto cada uno de los trabajadores de SOLCA del Cantón Jipijapa

Deductivo: En este método se observará los riesgos que pueden tener por la falta de conocimiento de las normas de seguridad e higiene ocupacional.

\section{TECNICAS}

Observación directa: Nos permito conocer la realidad de los riesgos de manera directa en la institución. 
Maryuri Yahaira Briones Mera, Fidel Alfredo Chonillo Toala, Jose Felix Peñafiel Loor...

Entrevista: Es un cuestionario que se dirigió al director de la Sociedad de Lucha contra el Cáncer del Cantón Jipijapa ya que él debe tener conocimiento de las normas de seguridad e higiene ocupacional que existen en la misma.

Encuesta: Se utilizó esta técnica para poder obtener información, clara, precisa y de esta manera llegar a cumplir con mi objetivo planteado.

\section{RESULTADOS}

\section{La importancia de la aplicación de las leyes y normativas de seguridad e higiene ocupacional}

La normativa vigente en el Ecuador en relación a la Seguridad e Higiene Ocupacional en el Ecuador se detalla a continuación con sus respectivos artículos:

El artículo 326, numeral 5, de la Constitución de la República del Ecuador establece que: "Toda persona tendrá derecho a desarrollar sus labores en un ambiente adecuado y propicio, que garantice la salud, integridad, seguridad, higiene y bienestar".

El artículo 326, numeral 6, de la Constitución de la República del Ecuador establece que: "Toda persona rehabilitada después de un accidente de trabajo o enfermedad, tendrá que ser reintegrado al trabajo y a mantener una relación laboral de acuerdo con la Ley". (Asamblea Nacional, 2008)

La constitución del Ecuador hace referencia a que toda persona que sea parte de una institución pública o privada tiene derecho a estar seguro al momento de desarrollar sus actividades en un ambiente sano y adecuado y también a que toda persona debe de ser reintegrada y mantener su relación laboral luego de ser rehabilitada por un accidente de trabajo o enfermedad.

En lo general

Todo empleador está obligado de garantizar un lugar de trabajo que no presenten peligros que puedan afectar la integridad física y la salud de sus trabajadores y por consiguiente los trabajadores estarán en la obligación de acatar y cumplir las medidas de prevención en seguridad e higiene brindadas por el empleador. Si algún trabajador llegase a incumplir la normativa el empleador tendrá derecho a dar por terminado el contrato de trabajo.

\section{Disminución de riesgos laborales}

La identificación de peligros, es la acción de observar, identificar y analizar los peligros o factores de riesgo, relacionados con los aspectos del trabajo, ambiente de trabajo, estructura e instalaciones, equipos de trabajo, maquinaria y herramientas.

Los peligros pueden ser químicos, físicos, biológico y factores de riesgo disergonómicos y psicosociales. La evaluación deberá realizarse considerando la información sobre la organización, 
las características y complejidad del trabajo, los materiales utilizados, los equipos existentes y el estado de salud de los trabajadores, valorando los riesgos existentes en función de criterios objetivos que brinden confianza sobre los resultados a alcanzar y para que el empleador se encuentre en condiciones de tomar decisiones apropiadas sobre la oportunidad, prioridad y tipo de acciones preventivas que debe adoptar.

"Artículo 4 del Capítulo II Política de Prevención de Riesgos Laborales establece que: "En el marco de sus sistemas nacionales de seguridad y salud en el trabajo, los países miembros deberán propiciar el mejoramiento de las condiciones de seguridad y salud en el trabajo, a fin de prevenir daños en la integridad física y mental de los trabajadores que sean consecuencia, guarden relación o sobrevengan durante el trabajo".

Artículo 11 del Capítulo III Gestión de la Seguridad y Salud en los centros de Trabajo Obligaciones de los Empleadores establece que: "En todo lugar de trabajo se deberán tomar medidas tendientes a disminuir los riesgos laborales. Estas medidas deberán basarse para el logro de este objetivo, en directrices de gestión de la seguridad y salud en el trabajo y su entorno como responsabilidad social y empresarial".

Artículo 12.- Los empleadores deberán adoptar y garantizar el cumplimiento de las medidas necesarias para proteger la salud y el bienestar de los trabajadores, entre otros, a través de los sistemas de gestión de seguridad y salud en el trabajo" (Baustista, 2000)

\section{CONCLUSIONES}

La institución carece de políticas internas establecidas basadas a la seguridad e higiene ocupacional. Así mismo, no disponen de una persona que este encargado de la prevención de riesgos laborales, a los cuales están expuestos los trabajadores.

Las autoridades, pocas veces socializan la normativa de seguridad e higiene ocupacional. Así mismo, existe poca importancia por parte de la administración, en cuanto a prevención de riesgos laborales, a los cuales se encuentran expuestos los trabajadores.

Es necesario comprender la importancia de la seguridad e higiene ocupacional. La alta competitividad de las instituciones en la actualidad las ha llevado a la necesidad de reconocer los peligros, abatir los riesgos y por ende los accidentes. Aun cuando se ha hablado de seguridad ocupacional y riesgos laborales desde hace mucho tiempo, podemos considerar que en el Ecuador es una disciplina muy joven, una prueba de ello, es que, la mayoría de las instituciones públicas o privadas no tienen programas formales de seguridad e higiene ocupacional y riesgo laboral.

\section{REFERÉNCIAS BIBLIOGRÁFICAS}

Asamblea Nacional. (2008). Constituciòn de la Republica del Ecuador. Montecristi: Registro Oficial.

Asociacion Española para la Calidad, A. (2013). AEC. Obtenido de Inspecciones de Seguridad Laboral: https://www.aec.es/web/guest/centro-conocimiento/inspecciones-de-seguridad-laboral

Baustista, R. (2000). Instrumento Andino de Seguridad y Salud en el Trabajo. Obtenido de Decisión CAN: http://www.industrias.ec/archivos/CIG/file/SEGURIDAD/DecisionCAN\%20584.pdf

Chiang, m., \& san martín, n. (12 de 2015). Análisis de la satisfacción y el desempeño laboral en los funcionarios de la municipalidad de talcahuano. Obtenido de https://scielo.conicyt.cl/scielo.php?pid=s071824492015000300001\&script=sci_arttext

Cortes, j. (2007). Seguridad e higiene en el trabajo: tecnicas de prevencion de riesgos laborales. En j. M. Diaz, seguridad e higiene en el trabajo: plan nacional y ordenanza general (pág. Pag. 45). Madrid: tebar,s.1.,. 
Maryuri Yahaira Briones Mera, Fidel Alfredo Chonillo Toala, Jose Felix Peñafiel Loor...

Grau, m. (2000). Seguridad $\quad$ laboral. $\quad$ Obtenido : http://webcache.googleusercontent.com/search?q=cache:rjw8dlvmvdqj:www.bvsde.paho.org/bvsacd/cd46/lsi_cap04. $\mathrm{pdf}+\& \mathrm{~cd}=6 \& \mathrm{hl}=\mathrm{es} \& \mathrm{ct}=\mathrm{clnk} \& \mathrm{gl}=\mathrm{ec}$

Haddad, I. R. (2013). Higiene y seguridad industrial. Obtenido de Objetivo de la Seguridad e Higiene: http://www.bvsde.paho.org/bvsacd/scan2/012335/012335-17.pdf

Hichez, r. (02 de 06 de 2013). Higiene, salud y seguridad ocupacional. Obtenido de http://www.eoi.es/blogs/madeon/2013/06/02/higiene-salud-y-seguridad-ocupacional/

Higiene y seguridad laboral. (martes de 09 de 2011). Disciplinas asociadas a la seguridad e higiene laborales desde la prevención. Obtenido de http://higieneysaludlaborales.blogspot.com/

Instituto de seguridad y servicios sociales de los trabajadores del estado. (2018). Seguridad e higiene en el trabajo - orientación de servicios y trámites de seguridad e higiene en el trabajo. Obtenido de https://www.gob.mx/issste/acciones-y-programas/seguridad-e-higiene-en-el-trabajo

Marben abogados. (2 de 05 de 2015). Seguridad e higiene en el trabajo. Obtenido de https://www.marbenabogados.com/seguridad-e-higiene-en-el-trabajo/

Mondy, w., noe, r., \& quiñones, a. (2005). Administración de recursos humanos. Colombia: person educacion de mexico s.a.

Navarrete, S. (2013). Scribd. Obtenido de Definición de Seguridad e Higiene Ocupacional: https://es.scribd.com/document/211373271/Capitulo-II

Navarro, m. (11 de 2014). El desempeño laboral y su incidencia en la calidad en el servicio en la cooperativa de ahorro y credito baños de agua http://repositorio.uta.edu.ec/bitstream/123456789/8410/1/205\%20mkt.pdf

Organización internacional del trabajo. (17 de 02 de 2014). Higiene ocupacional. Obtenido de http://empleosverdes.mex.ilo.org/trabajadores/la-importancia-de-la-higiene-industrial-en-los-trabajadores/

Prado, J. D. (26 de 10 de 2012). IMF Business School. Obtenido de Inspecciones de Seguridad: https://www.imfformacion.com/blog/prevencion-riesgos-laborales/actualidad-laboral/inspecciones-de-seguridad/

Rodriguez, E. (2010). Protección de la seguridad y salud de los trabajadores. Una revision desde la perspectiva global, latinoamericana y venezolana, 3

Velasco, F. (21 de septiembre de 2015). Blogger. Obtenido de Importancia de la Seguridad e Higiene: http://ruizvelascof.blogspot.com/2015/09/importancia-de-la-seguridad-e-higiene.html 